\title{
Using Homo-Separation of Variables for Solving Systems of Nonlinear Fractional Partial Differential Equations
}

\author{
Abdolamir Karbalaie, Hamed Hamid Muhammed, and Bjorn-Erik Erlandsson \\ Division of Informatics, Logistics and Management, School of Technology and Health (STH), Royal Institute of Technology (KTH), \\ 10044 Stockholm, Sweden \\ Correspondence should be addressed to Abdolamir Karbalaie; abdolamir.karbalaie@sth.kth.se
}

Received 18 March 2013; Accepted 24 May 2013

Academic Editor: Irena Lasiecka

Copyright (C) 2013 Abdolamir Karbalaie et al. This is an open access article distributed under the Creative Commons Attribution License, which permits unrestricted use, distribution, and reproduction in any medium, provided the original work is properly cited.

\begin{abstract}
A new method proposed and coined by the authors as the homo-separation of variables method is utilized to solve systems of linear and nonlinear fractional partial differential equations (FPDEs). The new method is a combination of two well-established mathematical methods, namely, the homotopy perturbation method (HPM) and the separation of variables method. When compared to existing analytical and numerical methods, the method resulting from our approach shows that it is capable of simplifying the target problem at hand and reducing the computational load that is required to solve it, considerably. The efficiency and usefulness of this new general-purpose method is verified by several examples, where different systems of linear and nonlinear FPDEs are solved.
\end{abstract}

\section{Introduction}

In the recent years, it has turned out that many phenomena in various technical and scientific fields can be described very successfully by using fractional calculus. In particular, fractional calculus can be employed to solve many problems within the biomedical research field and get better results. Such a practical application of fractional order models is to use these models to improve the behavior and efficiency of bioelectrodes. The importance of this application is based on the fact that bioelectrodes are usually needed to be used for all types of biopotential recording and signal measurement purposes such as electroencephalography (EEG), electrocardiography (ECG), and electromyography (EMG) [1-3]. Another promising biomedical application field is proposed by Arafa et al. [4] where a fractional-order model of HIV1 infection of $\mathrm{CD}^{+}{ }^{+} \mathrm{T}$ cells is introduced. Other examples of applications of fractional calculus in life sciences and technology can be found in botanics [5], biology [6], rheology [7], and elastography [8].

Numerous analytical methods have been presented in the literature to solve FPDEs, such as the fractional Greens function method [9], the Fourier transform method [2], the Sumudu transform method [10], the Laplace transform method, and the Mellin transform method [11]. Some numerical methods have also widely been used to solve systems of FPDEs, such as the variational iteration method [12], the Adomian decomposition method [2], the homotopy perturbation method [13] and the homotopy analysis method [14]. Some of these methods use specific transformations and others give the solution as a series which converges to the exact solution.

In addition, some numerical methods use a combination of utilizing specific transformations and obtaining series which converge to the exact solutions. An example of such a method is the iterative Laplace transform method which is a combination of the Laplace transform method and an iterative method [15]. Another such a combination is the homotopy perturbation transformation method, which is constructed by combining two powerful methods, namely, the Laplace transform method and the homotopy perturbation method [16]. A third example is the Sumudu decomposition method, which is a combination of the Sumudu transform method and Adomian decomposition method [17]. A fourth such an approach is combining the Sumudu transformation method with the homotopy perturbation 
method, which gives a new method called the homotopy perturbation Sumudu transform method [18].

Recently, the homotopy perturbation method and the Adomian decomposition method are frequently used for solving nonlinear FPDE problems.

According to the combinational methods mentioned above, it is possible to notice that HPM has a strong potential to be combined with another method to produce a more efficient approach. The main reason is that HPM is an efficient method for solving PDEs and ODEs (ordinary differential equations) with integer or fractional order. Recently, Karbalaie et al. [19] found the exact solution of one-dimensional FPDEs by using truncated versions of modified HPM. Therefore, by getting inspiration of the ideas, methods, and tools of previous works, the novel approach called the homoseparation of variables method is developed and utilized to find the exact solutions of systems of FPDEs. The methods presented in Karbalaie et al. [19] are extended and developed further to achieve methods that can solve n-dimensional systems of FPDEs. This new approach is constructed by a smart combination of HPM and the separation of variables method. By using this method, the system of FPDEs to be solved is changed into a system of FODEs. Consequently, the original problem is simplified considerably which makes it more straightforward and easier to solve.

The structure of the current paper is as follows. For easy reader-friendly reference, some basic definitions and properties of fractional differential equations are presented in Section 2. In Section 3, the homo-separation of variables method is described. After that, in Section 4, three examples are presented to show the simplicity and efficiency (at the same time) of the homo-separation of variables method. Finally, in Section 5, relevant conclusions are drawn.

\section{Basic Definitions and Properties}

In this section, to make it easier to introduce the new approach of this research work, a number of good-to-know concepts and properties within the field of fractional differential equations are defined and presented briefly.

Definition 1. A real function $f(t), t>0$, is said to be in the space $C_{\sigma}, \sigma \in \mathbb{R}$, if there exists a real number $p>\sigma$, such that $f(t)=t^{p} f_{1}(t)$, where $f_{1}(t) \in C[0, \infty)$, and it is said to be in the space $C_{\sigma}^{m}$ if $f^{m} \in C_{\sigma}, m \in \mathbb{N}$.

Definition 2. The left sided Riemann-Liouville fractional integral of order $\alpha \geq 0$, of a function $f \in C_{\sigma}, \sigma \geq-1$, is defined as

$$
J_{t}^{\alpha} f(t) \stackrel{\text { def }}{=} \frac{1}{\Gamma(\alpha)} \int_{0}^{t}(t-\tau)^{\alpha-1} f(\tau) d \tau
$$

where $\alpha>0, t>0$, and $\Gamma(\alpha)=\int_{0}^{\infty} r^{\alpha-1} e^{-r} d r$ is the gamma function.

Properties of the operator $J_{t}^{\alpha}$ (for $f \in C_{\mu}, \mu \geq-1, \alpha, \beta \geq$ $0, \gamma \geq-1)$ can be found in [20] and are defined as follows:

(1) $J_{t}^{0} f(t)=f(t)$,
(2) $J_{t}^{\alpha} J_{t}^{\beta} f(t)=J_{t}^{\alpha+\beta} f(t)$,

(3) $J_{t}^{\alpha} t^{\gamma}=(\Gamma(\gamma+1) / \Gamma(\alpha+\gamma+1)) t^{\alpha+\gamma}$.

Definition 3. The left sided Caputo fractional derivative of $f,\left(f \in C_{\mu}^{n}, \quad n \in \mathbb{N} \cup\{0\}\right)$, in Caputo sense is defined by [11] as follows:

$$
\begin{aligned}
& D_{t}^{\alpha} f(t) \\
& \stackrel{\text { def }}{=} \begin{cases}\frac{1}{\Gamma(n-\alpha)} \int_{0}^{t}(t-\tau)^{n-\alpha-1} f^{(n)}(\tau) d \tau, & n-1<\alpha \leq n, \\
D_{t}^{n} f(t), & \alpha=n .\end{cases}
\end{aligned}
$$

Note that, according to [21], (1) and (2) become

$$
\begin{gathered}
J_{t}^{\alpha} f(x, t)=\frac{1}{\Gamma(\alpha)} \int_{0}^{t}(t-\tau)^{\alpha-1} f(x, \tau) d \tau, \quad \alpha>0, t>0, \\
D_{t}^{\alpha} f(x, t)=\frac{1}{\Gamma(n-\alpha)} \int_{0}^{t}(t-\tau)^{n-\alpha-1} f^{(n)}(x, \tau) d \tau \\
n-1<\alpha \leq n .
\end{gathered}
$$

Definition 4. The single-parameter and the two-parameter variants of the Mittag-leffler function are denoted by $E_{\alpha}(t)$ and $E_{\alpha, \beta}(t)$, respectively. These two variants are relevant to be considered here because of their connection with fractional calculus and are defined as

$$
\begin{aligned}
E_{\alpha}(t) & =\sum_{j=0}^{\infty} \frac{t^{j}}{\Gamma(\alpha j+1)}, \quad \alpha>0, t \in \mathbb{C}, \\
E_{\alpha, \beta}(t) & =\sum_{j=0}^{\infty} \frac{t^{j}}{\Gamma(\alpha j+\beta)}, \quad \alpha, \beta>0, t \in \mathbb{C} .
\end{aligned}
$$

The $k$ th derivatives of these two variants are

$$
\begin{aligned}
E_{\alpha}^{(k)}(t) & =\frac{d^{k}}{d t^{k}} E_{\alpha}(t) \\
& =\sum_{j=0}^{\infty} \frac{(k+j) ! t^{j}}{j ! \Gamma(\alpha j+\alpha k+1)}, \quad k=0,1,2, \ldots, \\
E_{\alpha, \beta}^{(k)}(t) & =\frac{d^{k}}{d t^{k}} E_{\alpha, \beta}(t) \\
& =\sum_{j=0}^{\infty} \frac{(k+j) ! t^{j}}{j ! \Gamma(\alpha j+\alpha k+\beta)}, \quad k=0,1,2, \ldots .
\end{aligned}
$$

Some special cases and properties of the Mittag-Leffler functions are the following:

(1) $E_{\alpha, 1}(t)=E_{\alpha}(t)$,

(2) $E_{1,1}(t)=E_{1}(t)=e^{t}$,

(3) $E_{2,1}\left(t^{2}\right)=\cosh (t)$,

(4) $E_{2,2}\left(t^{2}\right)=\sinh (t) / t$, 
(5) $E_{\alpha, \beta}(t)+E_{\alpha, \beta}(-t)=2 E_{2 \alpha, \beta}\left(t^{2}\right)$,

(6) $E_{\alpha, \beta}(t)-E_{\alpha, \beta}(-t)=2 t E_{2 \alpha, \alpha+\beta}\left(t^{2}\right)$,

(7) $D_{t}^{\alpha}\left(t^{\alpha-1} E_{\alpha, \alpha}\left(a t^{\alpha}\right)\right)=t^{\alpha-1} E_{\alpha, \alpha}\left(a t^{\alpha}\right)$.

Further properties of the Mittag-Leffler functions can be found in [21]. These functions are generalizations of the exponential function; therefore, most linear differential equations of fractional order (FPDEs) have solutions that are expressed in terms of these functions.

Important Note. According to [22], $E_{\alpha}\left(a t^{\alpha}\right)$ cannot satisfy the following semigroup property:

$$
E_{\alpha}\left(a(t+s)^{\alpha}\right)=E_{\alpha}\left(a t^{\alpha}\right) E_{\alpha}\left(a s^{\alpha}\right), \quad t, s \geq 0, a \in \mathbb{R}
$$

for any noninteger $\alpha>0$. The above property is not true unless in the special cases where $\alpha=1$ or $=0$. Unfortunately, this misunderstanding can be found in a number of publications, which can be misleading and confusing.

Definition 5. Consider the following relaxation-oscillation equation, which is discussed in [23]:

$$
D_{t}^{\alpha} u(t)+A u(t)=f(t), \quad t>0,
$$

subject to the initial condition

$$
\left.\frac{\partial^{i}}{\partial t^{i}} u(t)\right|_{t=0}=u^{i}(0)=b_{i} \quad i=1,2, \ldots, n-1,
$$

where $b_{i}(i=1,2, \ldots, n-1)$ are real constants and $n-1<\alpha \leq$ $n$. The solution of (7) is obtained according to [23] as follows:

$$
\begin{aligned}
u(t)= & \int_{0}^{t}(t-\tau)^{\alpha-1} E_{\alpha, \alpha}\left(-A(t-\tau)^{\alpha}\right) f(\tau) d \tau \\
& +\sum_{j=0}^{n-1} b_{j} D_{t}^{\alpha-j-1}\left(t^{\alpha-1} E_{\alpha, \alpha}\left(-A t^{\alpha}\right)\right),
\end{aligned}
$$

where $E_{\alpha, \alpha}$ is the Mittag-Leffler function. It is easy to realize that if $0<\alpha \leq 1$, then the solution of (7) becomes as follows:

$$
\begin{aligned}
u(t)= & \int_{0}^{t}(t-\tau)^{\alpha-1} E_{\alpha, \alpha}\left(-A(t-\tau)^{\alpha}\right) f(\tau) d \tau \\
& +D_{t}^{\alpha-1}\left(t^{\alpha-1} E_{\alpha, \alpha}\left(-A t^{\alpha}\right)\right)
\end{aligned}
$$

which will be used to solve the systems of FPDEs in the examples discussed in this paper.

Definition 6. According to [24], consider the following linear system of fractional differential equations with its initial condition:

$$
\begin{gathered}
D_{t}^{\alpha} \mathbf{x}(t)=A \mathbf{x}(t), \quad 0<t \leq a, \\
\mathbf{x}(0)=\mathbf{x}_{0},
\end{gathered}
$$

where $\mathbf{x} \in \mathbb{R}^{n}, a>0$, the matrix $A \in \mathbb{R}^{n} \times \mathbb{R}^{n}$ and $D_{t}^{\alpha}$ is the Caputo fractional derivative of order $\alpha$, where $0<\alpha \leq 1$. The general analytical solution for (11) is given by

$$
\begin{aligned}
\mathbf{x}(t)= & c_{1} \mathbf{u}^{(1)} E_{\alpha}\left(\lambda_{1} t^{\alpha}\right)+c_{2} \mathbf{u}^{(2)} E_{\alpha}\left(\lambda_{2} t^{\alpha}\right) \\
& +\cdots+c_{n} \mathbf{u}^{(n)} E_{\alpha}\left(\lambda_{n} t^{\alpha}\right),
\end{aligned}
$$

where $c_{1}, c_{2}, \ldots, c_{n}$ are arbitrary constants, $E_{\alpha}$ is the MittagLeffler function, and $\lambda_{1}, \lambda_{2}, \ldots, \lambda_{n}$ and $\mathbf{u}^{(1)}, \mathbf{u}^{(2)}, \ldots, \mathbf{u}^{(n)}$ are the eigenvalues and the corresponding eigenvectors of the characteristic equation $\left(A-\lambda_{i} \mathbf{I}\right) \mathbf{u}^{(i)}=0$, where $i=1,2, \ldots, n$ and $\mathbf{I}$ is an $n \times n$ identity matrix.

\section{The Homo-Separation of Variables Method}

This approach is achieved by a novel combination of HPM and separation of variables. In this section, the algorithm of this new method is presented briefly by considering the following system of FPDEs:

$$
\begin{aligned}
D_{t}^{\alpha_{i}} u_{i}= & f_{i}(\bar{x}, t)+\mathscr{L}_{i}\left(u_{1}, u_{2}, \ldots, u_{n}\right) \\
& +\mathscr{N}_{i}\left(u_{1}, u_{2}, \ldots, u_{n}\right), \quad i=1,2, \ldots, n,
\end{aligned}
$$

subject to the initial conditions

$$
u_{i}(\bar{x}, 0)=g_{i}(\bar{x}),
$$

where $\bar{x} \in \mathbb{R}^{n-1}$, and for $i=1,2, \ldots, n$, the terms $\mathscr{L}_{i}$ represent linear operators, the terms $\mathcal{N}_{i}$ are nonlinear operators, while the terms $f_{i}$ represent known analytical functions. In addition, $D_{t}^{\alpha_{i}}=\partial^{\alpha_{i}} / \partial t^{\alpha_{i}}$ is the Caputo fractional derivative of order $\alpha_{i}$, where $0<\alpha_{i} \leq 1$ for $i=1,2, \ldots, n$.

According to the homotopy perturbation technique (HPM), we can construct the following homotopies:

$$
\begin{gathered}
(p-1)\left(D_{t}^{\alpha_{i}} V_{i}-D_{t}^{\alpha_{i}} u_{i 0}\right)+p\left(D_{t}^{\alpha_{i}} V_{i}-\widetilde{\mathscr{L}}_{i}-\widetilde{\mathscr{N}}_{i}-f_{i}(\bar{x}, t)\right) \\
=0, \quad i=1,2, \ldots, n,
\end{gathered}
$$

where $\widetilde{\mathscr{L}}_{i}=\mathscr{L}_{i}\left(V_{1}, V_{2}, \ldots, V_{n}\right), \widetilde{\mathcal{N}}_{i}=\mathcal{N}_{i}\left(V_{1}, V_{2}, \ldots, V_{n}\right)$, and the homotopy parameter, denoted by $p$, is considered as small $(p \in[0,1])$. In addition, for $i=1,2, \ldots, n, u_{i 0}=u_{i 0}(\bar{x}, t)$ is an initial approximation of the solution of (13) which satisfies the initial condition in (14).

We can assume that the solution of (15) can be expressed as a power series in $p$, as given below in (16).

$$
\begin{aligned}
& V_{1}=\sum_{j=0}^{\infty} p^{j} V_{1 j}=V_{10}+p V_{11}+p^{2} V_{12}+p^{3} V_{13}+\cdots, \\
& V_{2}=\sum_{j=0}^{\infty} p^{j} V_{2 j}=V_{20}+p V_{21}+p^{2} V_{22}+p^{3} V_{23}+\cdots,
\end{aligned}
$$

$$
V_{n}=\sum_{j=0}^{\infty} p^{j} V_{n j}=V_{n 0}+p V_{n 1}+p^{2} V_{n 2}+p^{3} V_{n 3}+\cdots
$$


By substituting (16) into (15) and equating the terms with identical powers of $p$, a set of equations is obtained as follows:

$$
\begin{gathered}
p^{0}: D_{t}^{\alpha_{i}} V_{i 0}-D_{t}^{\alpha_{i}} u_{i 0}=0, \\
p^{1}: D_{t}^{\alpha_{i}} V_{i 1}+D_{t}^{\alpha_{i}} u_{10} \\
-\mathscr{L}_{i}\left(V_{10}, V_{20}, \ldots, V_{n 0}\right)-\mathscr{M}_{i 0}-f_{i}=0, \\
\vdots \\
p^{j}: D_{t}^{\alpha_{i}} V_{i j}-\mathscr{L}_{i}\left(V_{1 j-1}, V_{2 j-1}, \ldots, V_{n j-1}\right)-\mathscr{M}_{i j-1}=0,
\end{gathered}
$$

where the terms $\mathscr{M}_{i j}(i=1,2, \ldots, n$ and $j=0,1, \ldots)$ are obtained from (17)-(19) by equating the coefficients of the nonlinear operators $\mathscr{N}_{i j}(i=1,2, \ldots, n$ and $j=0,1, \ldots)$ with the identical powers $j$ of $p$, that is, the terms containing $p^{j}$.

In case the $p$-parameter is considered as small, the best approximate solution of (13) can be readily obtained as follows:

$$
\begin{aligned}
& u_{i}(\bar{x}, t)=\lim _{p \rightarrow 1} \sum_{j=0}^{\infty} p^{j} V_{i j}(\bar{x}, t) \\
&= V_{i 0}(\bar{x}, t)+V_{i 1}(\bar{x}, t)+V_{i 2}(\bar{x}, t)+\cdots, \\
& i=1,2, \ldots, n,
\end{aligned}
$$

where $\bar{x}=\left(x_{1}, x_{2}, \ldots, x_{n-1}\right)$.

If in (20) there exists some $V_{i N}(\bar{x}, t)=0$, where $N \geq 1$ and $i=1,2, \ldots, n$, then the exact solution can be written in the following form:

$$
\begin{array}{r}
u_{i}(\bar{x}, t)=V_{i 0}(\bar{x}, t)+p V_{i 1}(\bar{x}, t)+\cdots+p^{N-1} V_{i N-1}(\bar{x}, t), \\
i=1,2, \ldots, n .
\end{array}
$$

For simplicity, we assume that $V_{i 1}(\bar{x}, t) \equiv 0$ in (21), which means that the exact solution in (13) is simplified as follows:

$$
u_{i}(\bar{x}, t)=V_{i 0}(\bar{x}, t), \quad i=1,2, \ldots, n .
$$

Now when solving (17), we obtain the following result:

$$
V_{i 0}(\bar{x}, t)=u_{i 0}(\bar{x}, t), \quad i=1,2, \ldots, n .
$$

By using (22) and (23) we have

$$
u_{i}(\bar{x}, t)=V_{i 0}(\bar{x}, t)=u_{i 0}(\bar{x}, t), \quad i=1,2, \ldots, n .
$$

The core of the new method proposed in this research work is to formulate the initial approximation of (13) in the form of separation of variables, as follows:

$$
\begin{aligned}
u_{i}(\bar{x}, t)= & u_{i 0}(\bar{x}, t)=u_{i}(\bar{x}, 0) c_{i 1}(t) \\
& +\left(\sum_{j=1}^{n} D_{x_{j}} u_{i}(\bar{x}, 0)\right) c_{i 2}(t) \\
= & g_{i}(\bar{x}) c_{i 1}(t)+\left(\sum_{j=1}^{n} D_{x_{j}} g_{i}(\bar{x})\right) c_{i 2}(t), \quad i=1,2, \ldots, n .
\end{aligned}
$$

The task now is to find the terms $c_{i 1}(t)$ and $c_{i 2}(t)$ to obtain the exact solution of the system of FPDEs in (13). Since (25) satisfies the initial conditions in (14), we get

$$
c_{i 1}(0)=1, \quad c_{i 2}(0)=0, \quad i=1,2, \ldots, n .
$$

By substituting (25) into (18), we get

$$
\begin{aligned}
D_{t}^{\alpha_{i}} V_{i 1}= & -D_{t}^{\alpha_{i}}\left(g_{i}(\bar{x}) c_{i 1}(t)+\left(\sum_{j=1}^{n} D_{x_{j}} g_{i}(\bar{x})\right) c_{i 2}(t)\right) \\
& -\mathbf{L}_{i}-\mathbf{M}_{i 0}+f_{i}(\bar{x}, t) \equiv 0,
\end{aligned}
$$

where $i=1,2, \ldots, n$ and

$$
\begin{array}{r}
\mathbf{L}_{i}=\mathscr{L}_{i}\left(g_{1}(\bar{x}) c_{11}(t)+\left(\sum_{j=1}^{n} D_{x_{j}} g_{1}(\bar{x})\right) c_{12}(t), \ldots,\right. \\
\left.\left(g_{n}(\bar{x}) c_{n 1}(t)+\left(\sum_{j=1}^{n} D_{x_{j}} g_{n}(\bar{x})\right) c_{n 2}(t)\right)\right), \\
\mathbf{M}_{i 0}=\mathscr{M}_{i 0}\left(g_{1}(\bar{x}) c_{11}(t)+\left(\sum_{j=1}^{n} D_{x_{j}} g_{1}(\bar{x})\right) c_{12}(t), \ldots,\right. \\
\left.\left(g_{n}(\bar{x}) c_{n 1}(t)+\left(\sum_{j=1}^{n} D_{x_{j}} g_{n}(\bar{x})\right) c_{n 2}(t)\right)\right) .
\end{array}
$$

Obviously, our goal is finally achieved and the system of FPDEs is changed into a system of FODEs. Consequently, the problem at hand is simplified considerably as it is well known that solving a system of FODEs is generally more straightforward and much easier than solving the corresponding system of FPDEs.

Finally, the target unknowns $c_{i 1}(t)$ and $c_{i 2}(t)$ can be obtained by utilizing and solving the system of FODEs and the initial conditions in (26).

\section{Applications}

In this section, we illustrate the applicability, simplicity, and efficiency of the homo-separation of variables method for solving systems of linear and nonlinear FPDEs.

Example 7. Consider the following system of linear FPDEs:

$$
\begin{aligned}
& D_{t}^{\alpha} u=v_{x}-v-u, \\
& D_{t}^{\alpha} v=u_{x}-v-u,
\end{aligned}
$$

where $0<\alpha \leq 1$, subject to the initial conditions

$$
u(x, 0)=\sinh (x), \quad v(x, 0)=\cosh (x) .
$$

To solve (29) and (30) by using the proposed homoseparation of variables method, we choose the initial approximations for (29) as follows:

$$
\begin{aligned}
& u_{0}(x, t)=u(x, 0) b_{1}(t)+D_{x} u(x, 0) b_{2}(t), \\
& v_{0}(x, t)=v(x, 0) c_{1}(t)+D_{x} v(x, 0) c_{2}(t) .
\end{aligned}
$$


By substituting the two initial conditions given by (30) into these two initial approximations, we get the following initial approximations:

$$
\begin{aligned}
& u_{0}(x, t)=b_{1}(t) \sinh (x)+b_{2}(t) \cosh (x), \\
& v_{0}(x, t)=c_{1}(t) \cosh (x)+c_{2}(t) \sinh (x) .
\end{aligned}
$$

By considering and following the approach which resulted in (27) and substituting the results presented by (32) into the system of linear FPDEs in (29), we get

$$
\begin{aligned}
D_{t} u_{1}= & \sinh (x)\left(D_{t}^{\alpha} b_{1}(t)+b_{1}(t)-c_{1}(t)+c_{2}(t)\right) \\
& +\cosh (x)\left(D_{t}^{\alpha} b_{2}(t)+b_{2}(t)+c_{1}(t)-c_{2}(t)\right) \equiv 0, \\
D_{t} v_{1}= & \cosh (x)\left(D_{t}^{\alpha} c_{1}(t)+c_{1}(t)-b_{1}(t)+b_{2}(t)\right) \\
& +\sinh (x)\left(D_{t}^{\alpha} c_{2}(t)+c_{2}(t)+b_{1}(t)-b_{2}(t)\right) \equiv 0 .
\end{aligned}
$$

By solving the latter system of linear FPDEs presented in (33), we obtain the following system of linear FODEs:

$$
\begin{aligned}
& D_{t}^{\alpha} b_{1}(t)+b_{1}(t)-c_{1}(t)+c_{2}(t)=0, \\
& D_{t}^{\alpha} b_{2}(t)+b_{2}(t)+c_{1}(t)-c_{2}(t)=0, \\
& D_{t}^{\alpha} c_{1}(t)+c_{1}(t)-b_{1}(t)+b_{2}(t)=0, \\
& D_{t}^{\alpha} c_{2}(t)+c_{2}(t)+b_{1}(t)-b_{2}(t)=0
\end{aligned}
$$

together with the initial conditions

$$
\begin{array}{ll}
b_{2}(0)=1, & b_{2}(0)=0, \\
c_{1}(0)=1, & c_{1}(0)=0 .
\end{array}
$$

By solving (34) and (35) by considering and applying Definition 6 which is discussed in Section 2, we obtain the following results:

$$
\begin{aligned}
& b_{1}(t)=\frac{1}{2}\left(E_{\alpha}\left(t^{\alpha}\right)+E_{\alpha}\left(-t^{\alpha}\right)\right), \\
& b_{2}(t)=\frac{1}{2}\left(-E_{\alpha}\left(t^{\alpha}\right)+E_{\alpha}\left(-t^{\alpha}\right)\right), \\
& c_{1}(t)=\frac{1}{2}\left(E_{\alpha}\left(t^{\alpha}\right)+E_{\alpha}\left(-t^{\alpha}\right)\right), \\
& c_{2}(t)=\frac{1}{2}\left(-E_{\alpha}\left(t^{\alpha}\right)+E_{\alpha}\left(-t^{\alpha}\right)\right) .
\end{aligned}
$$

By substituting (36) into (32) and applying Definition 4, in particular properties (2), (5) and (6) which are discussed in Section 2, we obtain the exact solution for the system of linear FPDEs in (29), as follows:

$$
\begin{aligned}
& u(x, t)=E_{2 \alpha, 1}\left(t^{2 \alpha}\right) \sinh (x)-t^{\alpha} E_{2 \alpha, \alpha+1}\left(t^{2 \alpha}\right) \cosh (x), \\
& v(x, t)=E_{2 \alpha, 1}\left(t^{2 \alpha}\right) \cosh (x)-t^{\alpha} E_{2 \alpha, \alpha+1}\left(t^{2 \alpha}\right) \sinh (x) .
\end{aligned}
$$

If we now put $\alpha \rightarrow 1$ in (37) or solve (29) and (30) for $\alpha=1$, we obtain the following exact solution for the corresponding system of linear PDEs:

$$
\begin{aligned}
& u(x, t)=\sinh (x-t), \\
& v(x, t)=\cosh (x-t) .
\end{aligned}
$$

Example 8. Consider the following system of inhomogeneous FPDEs:

$$
\begin{aligned}
& D_{t}^{\alpha} u+u_{x} v+u=1, \\
& D_{t}^{\beta} v-v_{x} u-v=1,
\end{aligned}
$$

where $0<\alpha$ and $\beta \leq 1$, subject to the initial conditions

$$
u(x, 0)=e^{x}, \quad v(x, 0)=e^{-x} .
$$

To solve (39) and (40) by using the new homo-separation of variables method, we choose the initial approximations for (39) as follows:

$$
\begin{aligned}
& u_{0}(x, t)=u(x, 0) c_{1}(t)+D_{x} u(x, 0) c_{2}(t), \\
& v_{0}(x, t)=v(x, 0) b_{1}(t)+D_{x} v(x, 0) b_{2}(t) .
\end{aligned}
$$

By substituting the two initial conditions given by (40) into these two initial approximations, we get the following initial approximations:

$$
\begin{gathered}
u_{0}(x, t)=e^{x} c_{1}(t)+e^{x} c_{2}(t), \\
v_{0}(x, t)=e^{-x} b_{1}(t)-e^{-x} b_{2}(t) .
\end{gathered}
$$

By considering and following the approach which resulted in (27) and substituting the results presented by (42) into the system of inhomogeneous FPDEs that we would like to solve, which is presented by (39), we get

$$
\begin{aligned}
D_{t}^{\alpha} u_{1}= & e^{x}\left(D_{t}^{\alpha}\left(c_{1}(t)+c_{2}(t)\right)+\left(c_{1}(t)+c_{2}(t)\right)\right) \\
& +\left(c_{1}(t)+c_{2}(t)\right)\left(b_{1}(t)-b_{2}(t)\right)-1 \equiv 0, \\
D_{t}^{\beta} v_{1}= & e^{x}\left(D_{t}^{\beta}\left(b_{1}(t)-b_{2}(t)\right)-\left(b_{1}(t)-b_{2}(t)\right)\right) \\
& +\left(c_{1}(t)+c_{2}(t)\right)\left(b_{1}(t)-b_{2}(t)\right)-1 \equiv 0 .
\end{aligned}
$$

By solving the latter system of FPDEs presented in (43), we obtain the following system of FODEs:

$$
\begin{gathered}
D_{t}^{\alpha}\left(c_{1}(t)+c_{2}(t)\right)+\left(c_{1}(t)+c_{2}(t)\right)=0, \\
D_{t}^{\beta}\left(b_{1}(t)-b_{2}(t)\right)-\left(b_{1}(t)-b_{2}(t)\right)=0, \\
\left(c_{1}(t)+c_{2}(t)\right)\left(b_{1}(t)-b_{2}(t)\right)-1=0
\end{gathered}
$$

together with the initial conditions

$$
\begin{array}{ll}
b_{1}(0)=1, & b_{2}(0)=0, \\
c_{1}(0)=1, & c_{2}(t)=0 .
\end{array}
$$


By solving (44) and (45) by considering and applying Definition 5 which is discussed in Section 2, we obtain the following results:

$$
\begin{gathered}
c_{1}(t)+c_{2}(t)=E_{\alpha}\left(-t^{\alpha}\right), \\
b_{1}(t)-b_{2}(t)=E_{\beta}\left(t^{\beta}\right), \\
E_{\alpha}\left(-t^{\alpha}\right) E_{\beta}\left(t^{\beta}\right)=1 .
\end{gathered}
$$

By substituting (46) into (42), we obtain the exact solution for the system of inhomogeneous FPDEs presented in (39), as follows:

$$
\begin{aligned}
& u(x, t)=e^{x} E_{\alpha}\left(-t^{\alpha}\right), \\
& v(x, t)=e^{-x} E_{\beta}\left(t^{\beta}\right), \\
& E_{\alpha}\left(-t^{\alpha}\right) E_{\beta}\left(t^{\beta}\right)=1 .
\end{aligned}
$$

Thus, this means that $u(x, t)=1 / v(x, t)$. If we now put $\alpha, \beta \rightarrow 1$ in (47), or solve (39) and (40) for $\alpha=\beta=1$, we obtain the following exact solution for the corresponding system of inhomogeneous PDEs:

$$
\begin{gathered}
u(x, t)=e^{x-t}, \\
v(x, t)=e^{-x+t} .
\end{gathered}
$$

Example 9. Consider the following system of nonlinear FPDEs:

$$
\begin{aligned}
& D_{t}^{\alpha} u=-u-v_{x} w_{y}-v_{y} w_{x}, \\
& D_{t}^{\beta} v=v-u_{x} w_{y}-u_{y} w_{x}, \\
& D_{t}^{\gamma} w=w-u_{x} v_{y}-u_{y} v_{x},
\end{aligned}
$$

where $0<\alpha$ and $\beta, \gamma \leq 1$, subject to the initial conditions

$$
\begin{gathered}
u(x, y, 0)=e^{x+y}, \quad v(x, y, 0)=e^{x-y}, \\
w(x, y, 0)=e^{-x+y} .
\end{gathered}
$$

To solve (49) and (50) by using the new homo-separation of variables method, we choose the initial approximations for (49) as follows:

$$
\begin{aligned}
u_{0}(x, y, t)= & u(x, y, 0) b_{1}(t) \\
& +\left(D_{x} u(x, y, 0)+D_{y} u(x, y, 0)\right) b_{2}(t), \\
v_{0}(x, y, t)= & v(x, y, 0) c_{1}(t) \\
& +\left(D_{x} v(x, y, 0)+D_{y} v(x, y, 0)\right) c_{2}(t), \\
w_{0}(x, y, t)= & w(x, y, 0) d_{1}(t) \\
& +\left(D_{x} w(x, y, 0)+D_{y} w(x, y, 0)\right) d_{2}(t) .
\end{aligned}
$$

By substituting the initial conditions given by (50) into these initial approximations, we get the following initial approximations:

$$
\begin{gathered}
u_{0}(x, y, t)=e^{x+y} b_{1}(t)+2 e^{x+y} b_{2}(t), \\
v_{0}(x, y, t)=e^{x-y} c_{1}(t), \\
w_{0}(x, y, t)=e^{-x+y} d_{1}(t) .
\end{gathered}
$$

By considering and following the approach which resulted in (27) and substituting the results presented by (52) into the system of nonlinear FPDEs that we would like to solve, which is presented by (49), we get

$$
\begin{gathered}
D_{t}^{\alpha} u_{1}=e^{-x+y}\left(D_{t}^{\alpha} b_{1}(t)+2 D_{t}^{\alpha} b_{2}(t)+b_{1}(t)+2 b_{2}(t)\right) \equiv 0, \\
D_{t}^{\beta} D_{t} v_{1}=e^{x-y}\left(D_{t}^{\beta} c_{1}(t)-c_{1}(t)\right) \equiv 0, \\
D_{t}^{\gamma} w_{1}=e^{-x+y}\left(D_{t}^{\gamma} d_{1}(t)-d_{1}(t)\right) \equiv 0 .
\end{gathered}
$$

By solving the latter system of FPDEs presented in (53), we obtain the following system of FODEs:

$$
\begin{gathered}
D_{t}^{\alpha}\left(b_{1}(t)+2 b_{2}(t)\right)+\left(b_{1}(t)+2 b_{2}(t)\right)=0, \\
D_{t}^{\beta} c_{1}(t)-c_{1}(t)=0, \\
D_{t}^{\gamma} d_{1}(t)-d_{1}(t)=0,
\end{gathered}
$$

together with the initial conditions

$$
\begin{array}{ll}
b_{1}(0)=1, & b_{2}(0)=0, \\
c_{1}(0)=1, & c_{2}(t)=0, \\
d_{1}(0)=1, & d_{2}(t)=0 .
\end{array}
$$

By solving (54) and (55) by considering and applying Definition 5 which is discussed in Section 2 of this paper, we obtain the following results:

$$
\begin{gathered}
b_{1}(t)-2 b_{2}(t)=E_{\alpha}\left(-t^{\alpha}\right), \\
c_{1}(t)=E_{\beta}\left(t^{\beta}\right), \\
d_{1}(0)=E_{\gamma}\left(t^{\gamma}\right) .
\end{gathered}
$$

By substituting (56) into (52), we obtain the exact solution for the system of nonlinear FPDEs in (49), as follows:

$$
\begin{gathered}
u(x, y, t)=e^{x+y} E_{\alpha}\left(-t^{\alpha}\right), \\
v(x, y, t)=e^{x-y} E_{\beta}\left(t^{\beta}\right), \\
w(x, y, t)=e^{-x+y} E_{\gamma}\left(t^{\gamma}\right) .
\end{gathered}
$$

If we now put $\alpha, \beta, \gamma \rightarrow 1$ in (57), or solve (49) and (50) for $\alpha=\beta=\gamma=1$, we obtain the following exact solution for the corresponding system of nonlinear PDEs:

$$
\begin{gathered}
u(x, y, t)=e^{x+y-t}, \\
v(x, y, t)=e^{x-y+t}, \\
w(x, y, t)=e^{-x+y+t} .
\end{gathered}
$$




\section{Conclusions}

In this paper, a novel approach was introduced and utilized to solve linear and nonlinear systems of FPDEs. The new technique was coined by the authors of this paper as the homo-separation of variables method. In this research work, it was demonstrated through different examples how this new method can be used for solving various systems of FPDEs.

When compared with the existing published methods, it is easy to notice that the new method has many advantages. It is straightforward, easy to understand, and fast, requiring much less computations to perform a limited number of steps of the simple procedure that can be applied to find the exact solution of a wide range of types of FPDE equations' systems. Furthermore, there is no need for using linearization or restrictive assumptions when employing this new method.

The basic principle of the current method is to perform an elegant combination (which is simple and smart) of two existing techniques. These two techniques are the popular homotopy perturbation method (HPM) that was originally proposed by $\mathrm{He}[25,26]$ and another popular approach called separation of variables. Finally, the resulting homoseparation of variables method, which is analytical, can be used to solve various systems of PDEs with integer and fractional order, for example, with respect to time.

\section{Acknowledgments}

The authors would like to thank Maryam Shabani and Mehran Shafaiee for their help and support as well as the inspiration the authors got through friendly scientific discussions.

\section{References}

[1] R. L. Magin, Fractional Calculus in Bioengineering, Begell House, West Redding, Conn, USA, 2006.

[2] R. L. Magin and M. Ovadia, "Modeling the cardiac tissue electrode interface using fractional calculus," JVC/Journal of Vibration and Control, vol. 14, no. 9-10, pp. 1431-1442, 2008.

[3] J. Keener and J. Sneyd, Mathematical Physiology, Springer, New York, NY, USA, 2004.

[4] A. A. M. Arafa, S. Z. Rida, and M. Khalil, "Fractional modeling dynamics of HIV and CD4+ T-cells during primary infection," Nonlinear Biomedical Physics, vol. 6, no. 1, article 1, 2012.

[5] I. S. Jesus, J. A. Tenreiro MacHado, and J. Boaventure Cunha, "Fractional electrical impedances in botanical elements," Journal of Vibration and Control, vol. 14, no. 9-10, pp. 1389-1402, 2008.

[6] K. S. Cole, "Sketches of general and comparative demography," in Cold Spring Harbor Symposia on Quantitative Biology, vol. 1, pp. 107-116, Cold Spring Harbor Laboratory Press, 1933.

[7] V. D. Djordjevi, J. Jari, B. Fabry, J. J. Fredberg, and D. Stamenovi, "Fractional derivatives embody essential features of cell rheological behavior," Annals of Biomedical Engineering, vol. 31, no. 6, pp. 692-699, 2003.

[8] C. Coussot, S. Kalyanam, R. Yapp, and M. Insana, "Fractional derivative models for ultrasonic characterization of polymer and breast tissue viscoelasticity," IEEE Transactions on Ultrasonics, Ferroelectrics, and Frequency Control, vol. 56, no. 4, pp. 715725, 2009.

[9] F. Mainardi, "Fractional diffusive waves in viscoelastic solids," in Nonlinear Waves in Solids, pp. 93-97, Fairfield, 1995.

[10] V. G. Gupta and B. Sharma, "Application of Sumudu transform in reaction-diffusion systems and nonlinear waves," Applied Mathematical Sciences, vol. 4, no. 9-12, pp. 435-446, 2010.

[11] I. Podlubny, Fractional Differential Equations: An Introduction to Fractional Derivatives, Fractional Differential Equations, to Methods of Their Solution and Some of Their Applications, vol. 198 of Mathematics in Science and Engineering, Academic Press, San Diego, Calif, USA, 1999.

[12] S. Momani and Z. Odibat, "Numerical approach to differential equations of fractional order," Journal of Computational and Applied Mathematics, vol. 207, no. 1, pp. 96-110, 2007.

[13] O. Abdulaziz, I. Hashim, and S. Momani, "Solving systems of fractional differential equations by homotopy-perturbation method," Physics Letters A, vol. 372, no. 4, pp. 451-459, 2008.

[14] M. Ganjiani and H. Ganjiani, "Solution of coupled system of nonlinear differential equations using homotopy analysis method," Nonlinear Dynamics of Nonlinear Dynamics and Chaos in Engineering Systems, vol. 56, no. 1-2, pp. 159-167, 2009.

[15] H. Jafari, M. Nazari, D. Baleanu, and C. M. Khalique, "A new approach for solving a system of fractional partial differential equations," Computers \& Mathematics with Applications, 2012.

[16] S. Kumar, A. Yildirim, and L. Wei, "A fractional model of the diffusion equation and its analytical solution using Laplace transform," Scientia Iranica, vol. 19, no. 4, pp. 1117-1123, 2012.

[17] D. Kumar, J. Singh, and S. Rathore, "Sumudu decomposition method for nonlinear equations," International Mathematical Forum, vol. 7, no. 9-12, pp. 515-521, 2012.

[18] J. Singh, D. Kumar, and Sushila, "Homotopy perturbation sumudu transform method for nonlinear equations," Advances in Theoretical and Applied Mechanics, vol. 4, no. 4, pp. 165-175, 2011.

[19] A. Karbalaie, M. M. Montazeri, and H. H. Muhammed, "New approach to find the exact solution of fractional partial differential equation," WSEAS Transactions on Mathematics, vol. 11, no. 10, pp. 908-917, 2012.

[20] S. G. Samko, A. A. Kilbas, and O. I. Marichev, Fractional Integrals and Derivatives: Theory and Applications, Gordon and Breach Science Publishers, Yverdon, Switzerland, 1993.

[21] A. A. M. Arafa, S. Z. Rida, and H. Mohamed, "Homotopy analysis method for solving biological population model," Communications in Theoretical Physics, vol. 56, no. 5, pp. 797800, 2011.

[22] J. Peng and K. Li, "A note on property of the Mittag-Leffler function," Journal of Mathematical Analysis and Applications, vol. 370, no. 2, pp. 635-638, 2010.

[23] J.-F. Cheng and Y.-M. Chu, "Solution to the linear fractional differential equation using Adomian decomposition method," Mathematical Problems in Engineering, vol. 2011, Article ID 587068, 14 pages, 2011.

[24] Z. M. Odibat, "Analytic study on linear systems of fractional differential equations," Computers \& Mathematics with Applications, vol. 59, no. 3, pp. 1171-1183, 2010.

[25] J. He, "Application of homotopy perturbation method to nonlinear wave equations," Chaos, Solitons \& Fractals, vol. 26, no. 3, pp. 695-700, 2005. 
[26] J. H. He, "Homotopy perturbation method for bifurcation of nonlinear problems," International Journal of Nonlinear Sciences and Numerical Simulation, vol. 6, pp. 207-208, 2005. 


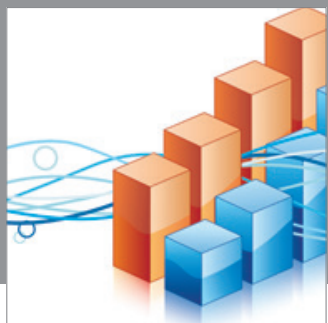

Advances in

Operations Research

mansans

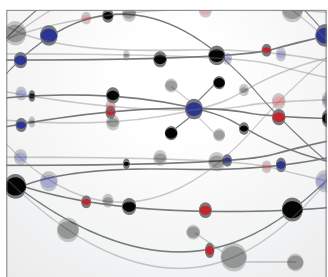

The Scientific World Journal
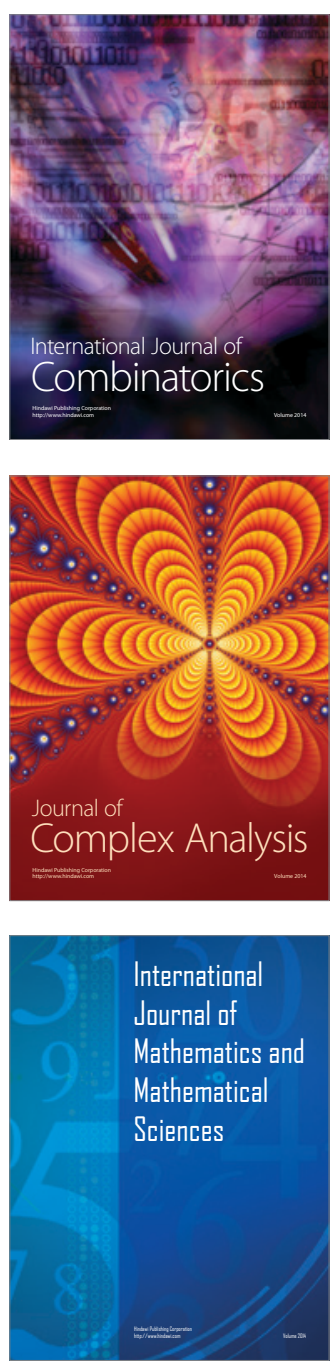
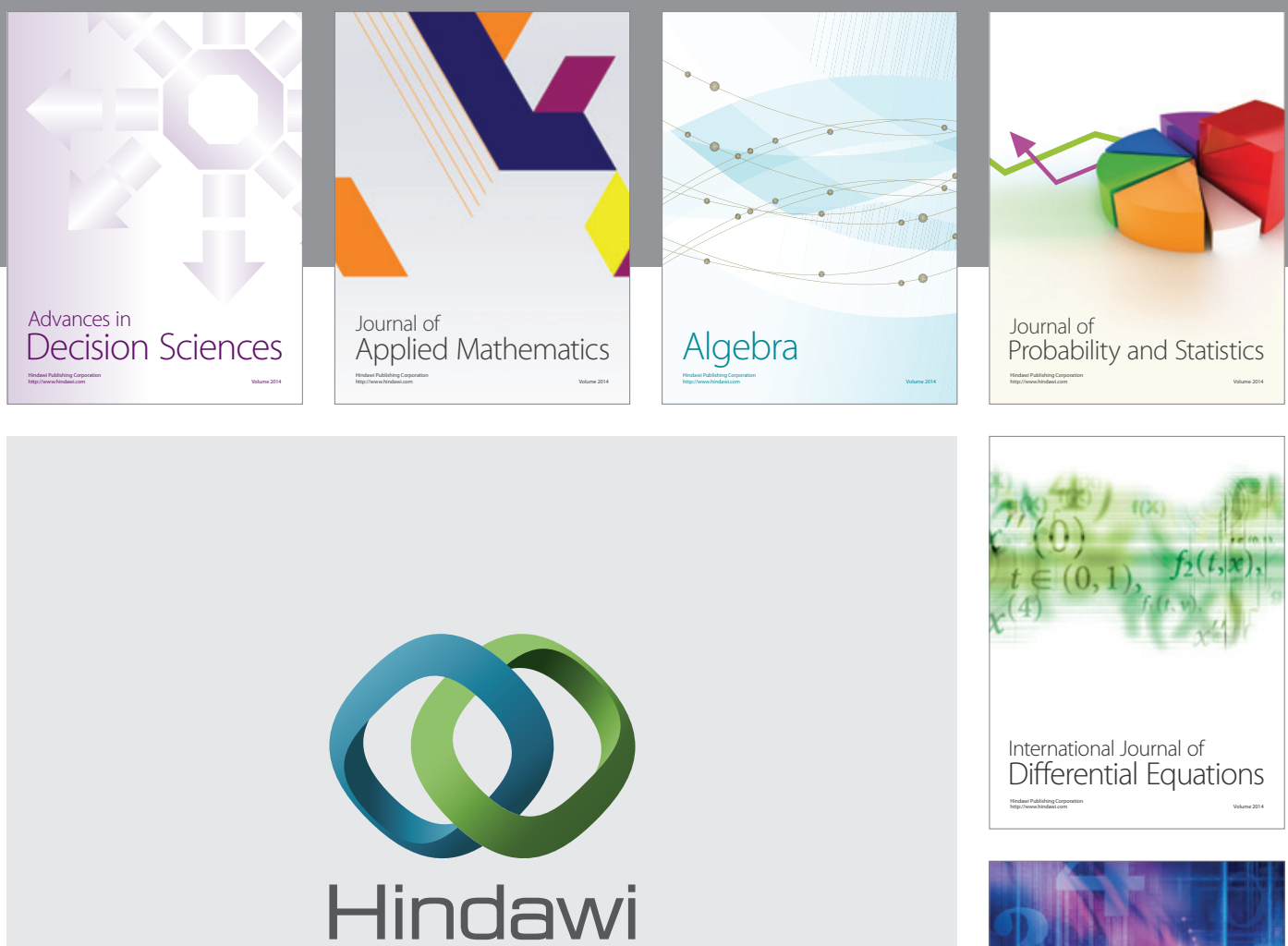

Submit your manuscripts at http://www.hindawi.com
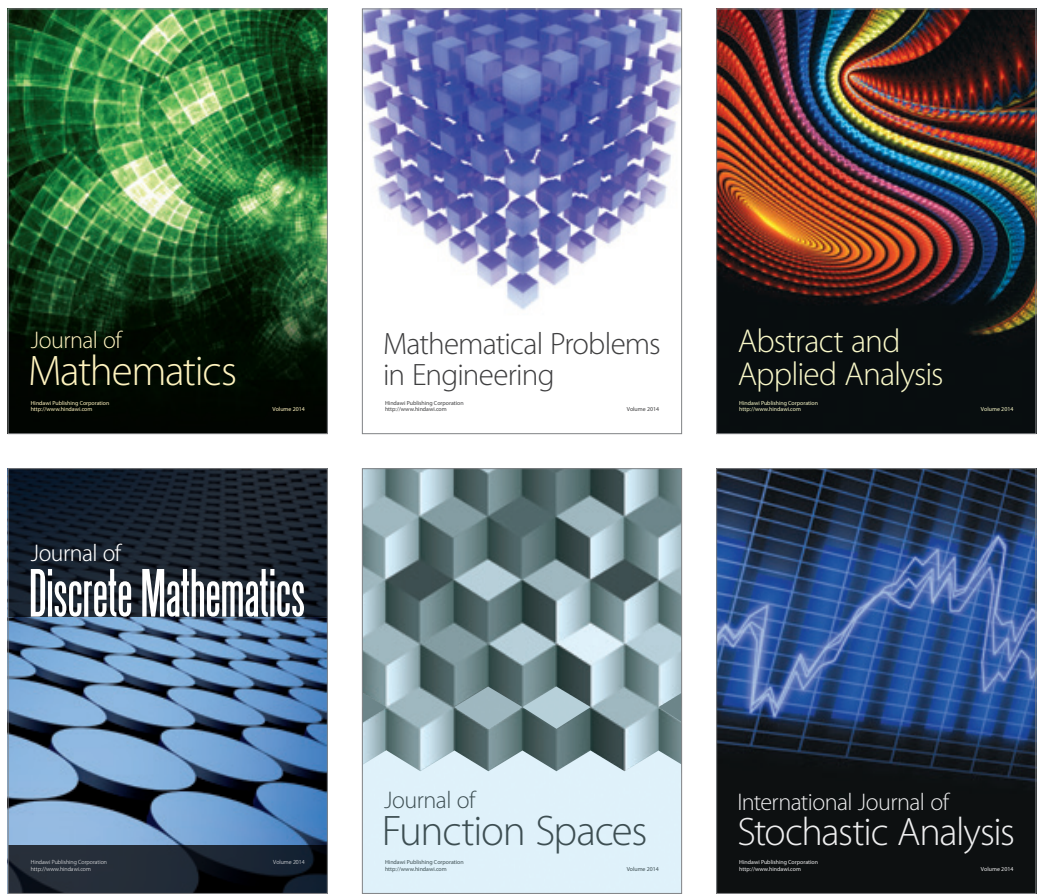

Journal of

Function Spaces

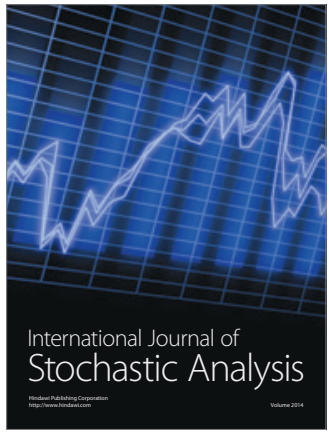

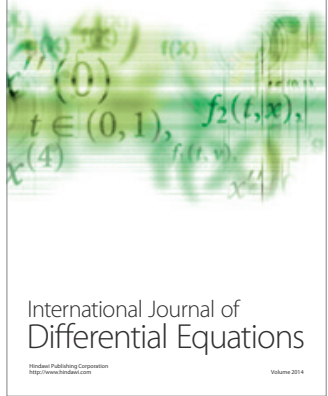
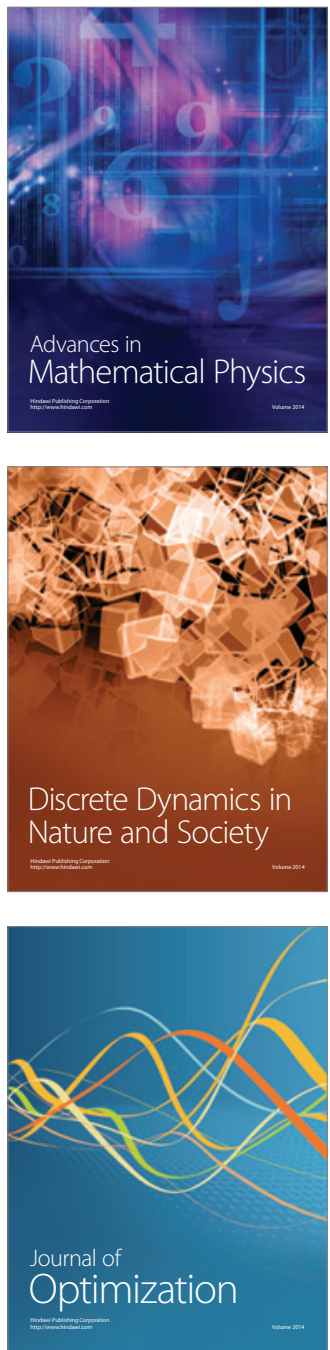